\title{
TRANSVERSE OSCILLATIONS IN CHROMOSPHERIC MOTTLES
}

\author{
D. Kuridze ${ }^{1,2}$, R. J. Morton ${ }^{3}$, R. Erdélyi ${ }^{3}$, G. D. Dorrian ${ }^{4}$, M. Mathioudakis ${ }^{1}$, D. B. Jess ${ }^{1}$, And F. P. Keenan ${ }^{1}$ \\ ${ }^{1}$ Astrophysics Research Centre, School of Mathematics and Physics, Queen's University, Belfast BT7 1NN, UK; dkuridze01@qub.ac.uk \\ ${ }^{2}$ Abastumani Astrophysical Observatory, Ilia State University, G. Tsereteli 3, 0612, Tbilisi, Georgia \\ ${ }^{3}$ Solar Physics and Space Plasma Research Centre (SP2 RC), University of Sheffield, Hicks Building, Hounsfield Road, Sheffield S3 7RH, UK \\ ${ }^{4}$ Institute of Astronomy and Astrophysics, National Observatory of Athens, Lofos Nymfon, Thiseio, P.O. Box 20048, GR-11810 Athens, Greece \\ Received 2012 January 10; accepted 2012 February 24; published 2012 April 13
}

\begin{abstract}
A number of recent investigations have revealed that transverse waves are ubiquitous in the solar chromosphere. The vast majority of these have been reported in limb spicules and active region fibrils. We investigate long-lived, quiet-Sun, on-disk features such as chromospheric mottles (jet-like features located at the boundaries of supergranular cells) and their transverse motions. The observations were obtained with the Rapid Oscillations in the Solar Atmosphere instrument at the Dunn Solar Telescope. The data set is comprised of simultaneous imaging in the $\mathrm{H} \alpha$ core, $\mathrm{Ca}$ II $\mathrm{K}$, and $G$ band of an on-disk quiet-Sun region. Time-distance techniques are used to study the characteristics of the transverse oscillations. We detect over 40 transverse oscillations in both bright and dark mottles, with periods ranging from 70 to $280 \mathrm{~s}$, with the most frequent occurrence at $\sim 165 \mathrm{~s}$. The velocity amplitudes and transverse displacements exhibit characteristics similar to limb spicules. Neighboring mottles oscillating in-phase are also observed. The transverse oscillations of individual mottles are interpreted in terms of magnetohydrodynamic kink waves. Their estimated periods and damping times are consistent with phase mixing and resonant mode conversion.
\end{abstract}

Key words: magnetohydrodynamics (MHD) - Sun: atmosphere - Sun: chromosphere - Sun: oscillations magnetic fields - waves

Online-only material: color figures

\section{INTRODUCTION}

Dark and bright mottles are commonly observed in the quiescent solar chromosphere. They are small-scale jet-like features of relatively cool and dense material located at the boundaries of supergranular cells, ejected from the lower chromosphere at speeds of about $10-40 \mathrm{~km} \mathrm{~s}^{-1}$ (Tsiropoula \& Tziotziou 2004; Rouppe van der Voort et al. 2007). A number of earlier studies suggest that bright and dark mottles correspond to phenomena in the lower and upper chromospheres, respectively (Bray 1969), while others believe that the difference in brightness indicates different parts of the same structure (Beckers 1968, 1972; Banos \& Macris 1970; Bray \& Loughhead 1974; Sterling et al. 1993). Mottles are often considered as the disk representation of chromospheric spicules (Hansteen et al. 2006; Rouppe van der Voort et al. 2009). The exact nature of mottles remains the subject of an ongoing debate, with the majority of solar researchers agreeing that mottles and spicules are related, in the sense that they have similar temperatures, density profiles, widths, lengths, and lifetimes (Tsiropoula \& Schmieder 1997; Zachariadis et al. 2001). Chromospheric small-scale jet-like structures, such as mottles, spicules, and fibrils, play an important role in the mass balance of the solar atmosphere. It is estimated that only a small percentage of the mass outflow provided by mottles is sufficient to compensate for the coronal mass loss due to the solar wind (Tsiropoula \& Tziotziou 2004).

The highly dynamic photosphere can excite magnetohydrodynamic (MHD) waves which can propagate into the chromosphere and corona (Erdélyi 2006a, 2006b). Spicular structures can act as conduits for transferring wave energy from the lower to the upper parts of the solar atmosphere. Numerous observations of transverse motions in spicular structures have been reported in recent years (Kukhianidze et al. 2006; De Pontieu et al. 2007; He et al. 2009b; Tavabi et al. 2011; Verth et al. 2011;
Okamoto \& de Pontieu 2011). An excellent review on the topic is given by Zaqarashvili \& Erdélyi (2009). Kink waves have also been recently observed in active region fibrils (Pietarila et al. 2011). These are often considered as on-disk, active region spicules that connect areas of opposite magnetic polarity (van Noort \& Rouppe van der Voort 2006; De Pontieu et al. 2007; Guglielmino et al. 2010; Kuridze et al. 2011). Spicules and mottles are typically short-lived features, with lifetimes of the order of typical wave periods. The observed waves also appear to propagate with phase speeds close to the local Alfvén speed. Mottles and spicules have typical lengths of 4-6 Mm, so waves propagate along the mottle in only $60-90 \mathrm{~s}$. Both of these features make it challenging to obtain information on the observed wave motion, e.g., amplitudes, periods, phase speeds. However, the reported wave periods in spicules and fibrils are in the range of 1-5 minutes. Transverse motions in spicules have, in the past, been misinterpreted as Alfvén waves, yet spicules (and mottles) appear as overdense structures in observations that are assumed to outline the quiet-Sun magnetic field. In this case, the pure Alfvén waves are torsional motions (Erdélyi \& Fedun 2007) and the transverse waves are the MHD kink modes.

Here, we present high spatial and temporal resolution observations of transverse oscillations in on-disk bright and dark mottles in the quiet-solar chromosphere. We focus our attention on relatively long-lived mottles that display transverse motions which last for at least one wave cycle. We identify over 40 such waves and are able to obtain statistical information on the wave properties (e.g., amplitudes, periods).

\section{OBSERVATIONS AND DATA REDUCTION}

The observations were obtained between 15:41 and 16:51 UT on 2010 September 29 with the Rapid Oscillations in the Solar Atmosphere (ROSA; Jess et al. 2010) imaging system, mounted 

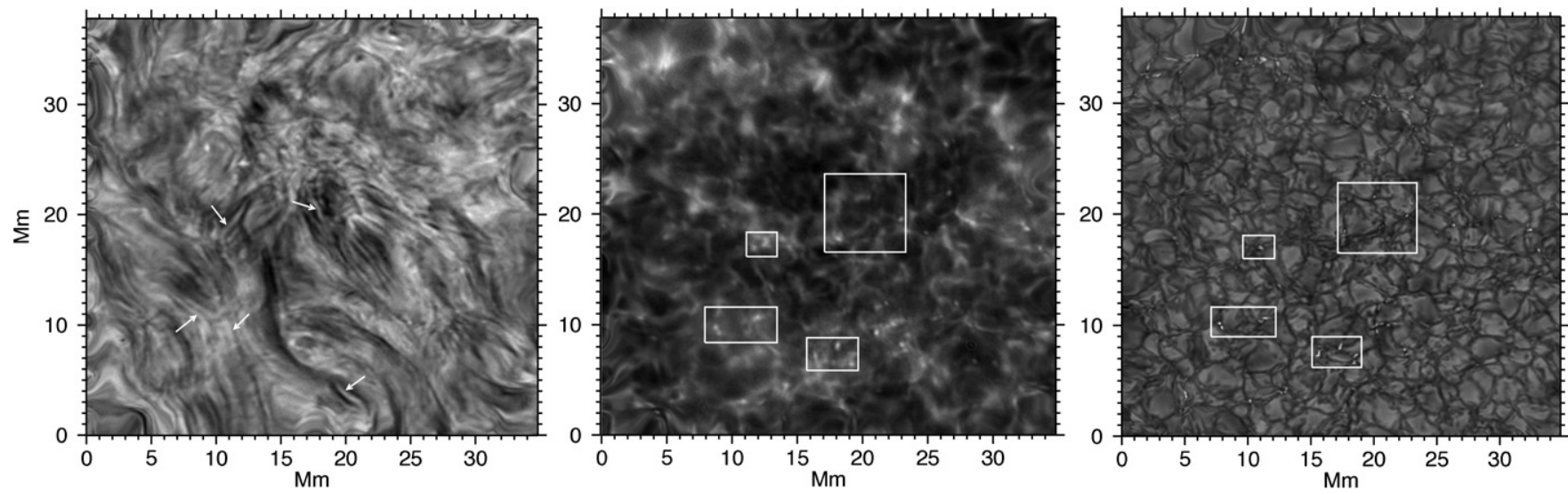

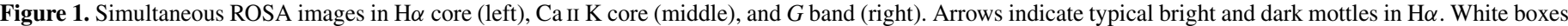
indicate areas in which long-lived mottles have been detected in $\mathrm{H} \alpha$.
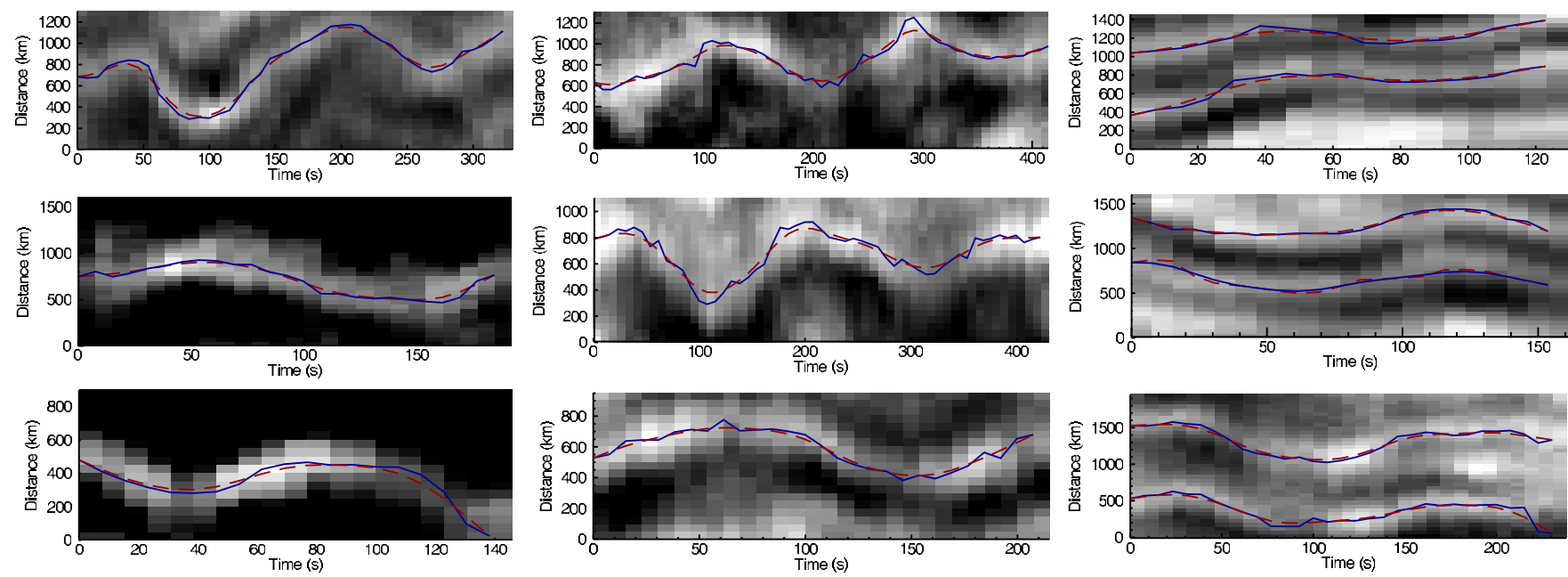

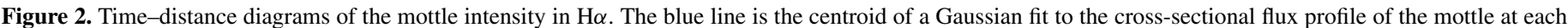
time step smoothed by a $\sim 50 \mathrm{~km}$ width (red line).

(A color version of this figure is available in the online journal.)

on the Dunn Solar Telescope (DST) at the National Solar Observatory, New Mexico, USA. A typical data set includes simultaneous imaging in $G$ band, $\mathrm{H} \alpha$ core, and $\mathrm{Ca}$ II K. Highorder adaptive optics were used throughout the observations. The images were reconstructed using the algorithms of Wöger et al. (2008) followed by de-stretching. These algorithms were implemented to remove the effects of atmospheric distortion from the data. The effective cadence after reconstruction was $7.7 \mathrm{~s}$ for $\mathrm{H} \alpha, 9.6 \mathrm{~s}$ for $\mathrm{Ca} \mathrm{II} \mathrm{K}$, and $1 \mathrm{~s}$ for the $G$ band. The total field of view is $48^{\prime \prime} \times 52^{\prime \prime}$, centered at $\sim 15^{\prime \prime}, 0^{\prime \prime}$, with a spatial sampling of 0.069 pixel $^{-1}$ corresponding to a spatial resolution of $150 \mathrm{~km}$ in $\mathrm{H} \alpha$.

\section{ANALYSIS AND DISCUSSION}

Figure 1 shows co-spatial and co-temporal images of the field of view in the $\mathrm{H} \alpha$ core, $\mathrm{Ca}$ II $\mathrm{K}$, and $G$ band. The $\mathrm{H} \alpha$ image is dominated by elongated dark and bright mottles which appear co-spatial with $\mathrm{Ca}$ II $\mathrm{K}$ brightenings and photospheric magnetic bright points.

We find a total of 42 mottles (23 bright, 19 dark) that show transverse waves lasting for at least one wave cycle. In seven cases, neighboring mottles are seen to oscillate in phase. Time-distance analysis reveals transverse oscillatory motions perpendicular to the mottle axis. The center is determined by fitting a Gaussian profile to the cross-sectional flux profile for each time frame of the transverse cut (blue lines in Figure 2). The overall motion of the mottle shows a linear trend which has been subtracted from the original signal. Oscillation periods were determined using wavelet analysis (Torrence \& Compo 1998) and found to vary between 70 and $280 \mathrm{~s}$, with a strong peak at $\sim 165 \mathrm{~s}$. Transverse velocities are in the range of $3-18 \mathrm{~km} \mathrm{~s}^{-1}$, with displacement amplitudes between 100 and $400 \mathrm{~km}$ (Figure 3).

The phase speed of the transverse motions can be evaluated by determining the phase difference between signals of the waves obtained at different heights along the mottle. Phase difference analysis along the mottle axis can be undertaken accurately only for seven mottles due to the complex fine structure of the $\mathrm{H} \alpha$ images. Estimated phase speeds are in the range of $40-110 \mathrm{~km} \mathrm{~s}^{-1}$. In most cases the oscillations are seen only in a segment of the mottle typically less than $1 \mathrm{Mm}$ long. A cadence of $\sim 7.7 \mathrm{~s}$ limits the maximum phase speed that may be detected to $\sim 130 \mathrm{~km} \mathrm{~s}^{-1}$. The mean period, amplitude, and phase speed of the observed oscillations are similar to results found for limb spicules (Kukhianidze et al. 2006; Zaqarashvili et al. 2007; Kim et al. 2008; He et al. 2009b; Okamoto \& de Pontieu 2011).

Mottles can be considered as straight, cylindrical, highdensity magnetic flux tubes which can guide different types 

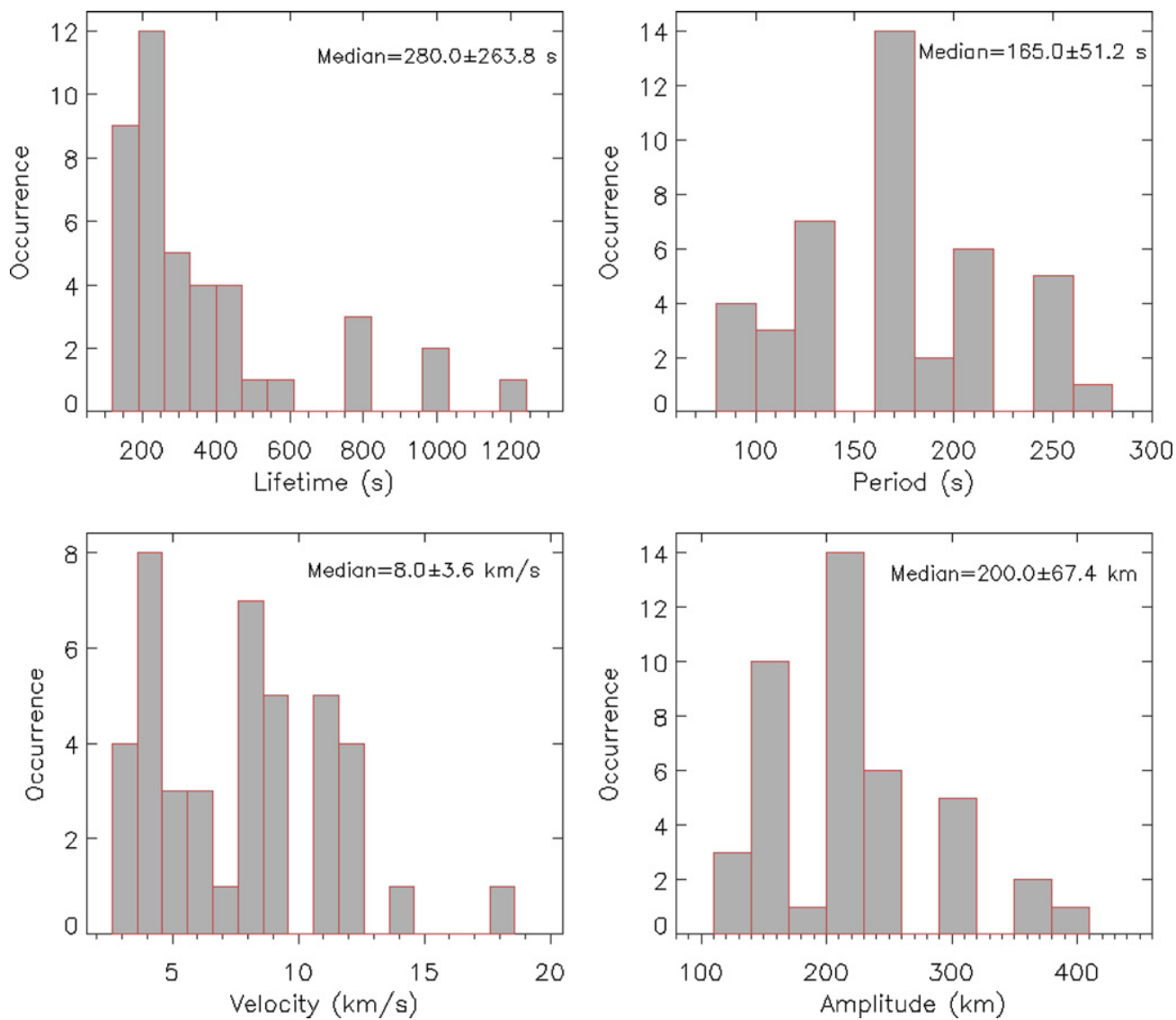

Figure 3. Lifetime (top left), period (top right), transverse velocity amplitude (bottom left), and transverse displacement amplitude (bottom right) for the 42 mottles analyzed in this study. Median values with standard deviations are also given.

(A color version of this figure is available in the online journal.)

of MHD wave modes, e.g., slow, fast, and Alfvén. Alfvén waves, propagating incompressibly along a magnetic flux tube, are torsional and do not displace the tube axis. MHD sausage modes may lead to intensity variations or variations of the mottle cross-section but do not cause a transverse displacement of the tube axis. The clear transverse displacement of the mottle axis seen in our observations suggests that the most appropriate interpretation for the oscillations is that of propagating/standing kink waves. The characteristic speed for the transverse motions is the kink speed, $c_{k}$, defined as (Edwin \& Roberts 1983)

$$
c_{k}^{2}=\frac{\rho_{0} v_{A 0}^{2}+\rho_{e} v_{A e}^{2}}{\rho_{0}+\rho_{e}},
$$

where $\rho_{0}, v_{A 0}, \rho_{e}$, and $v_{A e}$ are the plasma densities and Alfvén speeds inside and outside the tube, respectively. This equation is applicable near the thin tube limit, i.e., $r \ll \lambda$, where $\lambda$ and $r$ are the wavelength and mottle radius, respectively. The wavelength, $\lambda$, of the transverse wave is given by $\lambda=T c_{k}$, where $T$ is the period of the wave. Hence $\lambda=4800 \mathrm{~km}$ for $T=80 \mathrm{~s}$ and $c_{k}=60 \mathrm{~km} \mathrm{~s}^{-1}$. The radius of a mottle is typically $\sim 200 \mathrm{~km}$, hence $r \ll \lambda$, the thin tube limit applies, and phase speed can be approximated by the kink speed. Furthermore, mottles are cool and dense material in the vicinity of strong magnetic flux concentration in network regions co-spatial with photospheric magnetic bright points (Figure 1). They may therefore be considered as low- $\beta$ plasma magnetic tubes embedded in the field free (non magnetic) or weak field (compared to the mottle itself) environment. For no magnetic field outside the tube,
Equation (1) implies that

$$
c_{k}=\sqrt{\frac{\rho_{0}}{\rho_{0}+\rho_{e}}} v_{A 0}=\sqrt{\frac{1}{1+\rho_{e} / \rho_{0}}} \frac{B_{0}}{\sqrt{4 \pi \rho_{0}}},
$$

where $\mathrm{B}_{0}$ is the tube magnetic field strength. For a typical value of the tube magnetic field $B_{0} \approx 10 \mathrm{G}$ (Trujillo Bueno et al. 2005), density $\rho_{0} \approx(1-3) \times 10^{-13} \mathrm{~g} \mathrm{~cm}^{-3}$ (Beckers 1972; Sterling 2000; Tsiropoula \& Tziotziou 2004), and $\rho_{e} / \rho_{0} \sim$ $0.1-0.033$, we estimate from Equation (2) kink speed range of $\sim 46-86 \mathrm{~km} \mathrm{~s}^{-1}$. This value compares well with our measured phase speeds $\left(\sim 40-110 \mathrm{~km} \mathrm{~s}^{-1}\right)$. The observed periods show a strong peak at around $165 \mathrm{~s}$ (top right panel of Figure 3). This value is very close to the chromospheric kink wave cutoff period defined as $T_{k}=8 H \pi / c_{k}$, where $H$ is the chromospheric density scale height (Rae \& Roberts 1982; Spruit \& Roberts 1983; Roberts 2004). For the kink speed discussed above and a chromospheric scale height of $H \sim 500 \mathrm{~km}$, we estimate a kink wave cutoff period of $T_{k} \sim 170 \mathrm{~s}$. Waves with periods above the cutoff become evanescent. However, wave propagation in non-ideal (e.g., with ion-neutral collisions or non-adiabatic effects) or inclined magnetic tubes can lead to an increase in the cutoff period and allows the penetration of lower-frequency oscillations higher up in the solar atmosphere (De Pontieu et al. 2004). Our imaging observations do not allow us to define accurate inclination angles for individual mottles. However, as we are looking at the projection of mottle lengths onto the plane of the sky, this implies that they are inclined from the vertical. 

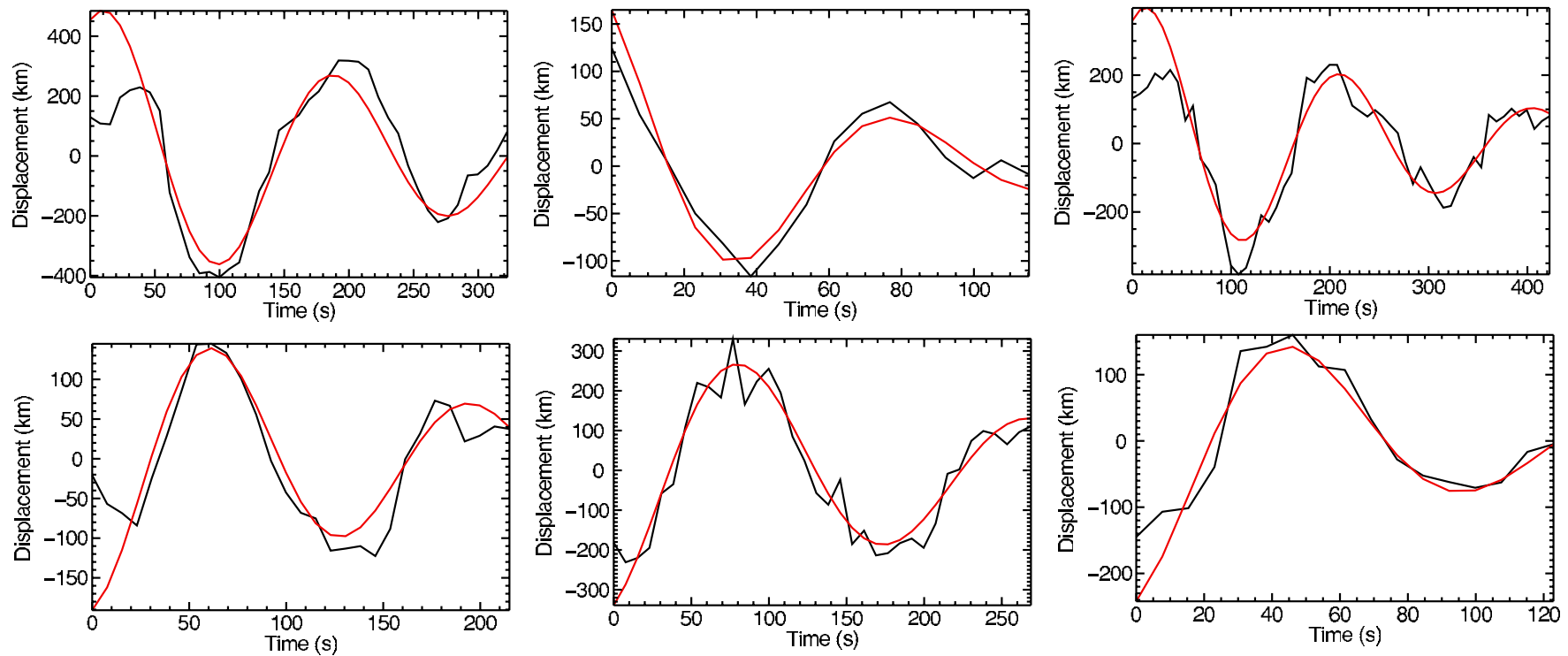

Figure 4. Observed mottle displacements (black line) fitted with an exponentially damped cosine function (red line).

(A color version of this figure is available in the online journal.)

Table 1

The Amplitude $A$, Oscillation Period $T$, Damping Time $\tau$, and Phase $\phi$ for the Damped Oscillations

\begin{tabular}{lcccc}
\hline \hline $\begin{array}{l}\text { Damping } \\
\text { Cases }\end{array}$ & $A$ & $T$ & $\tau$ & $\phi$ \\
& $(\mathrm{km})$ & $(\mathrm{s})$ & $(\mathrm{s})$ & $0.45 \pm 0.15$ \\
1 & $400 \pm 75$ & $165 \pm 12$ & $285 \pm 70$ & $0.42 \pm 0.22$ \\
2 & $150 \pm 50$ & $80 \pm 5$ & $62 \pm 40$ & $0.54 \pm 0.1$ \\
3 & $300 \pm 65$ & $190 \pm 5$ & $294 \pm 30$ & $2.8 \pm 0.05$ \\
4 & $330 \pm 80$ & $170 \pm 17$ & $263 \pm 75$ & $3 \pm 0.5$ \\
5 & $170 \pm 60$ & $120 \pm 12$ & $191 \pm 35$ & $3 \pm 0.1$ \\
6 & $150 \pm 50$ & $80 \pm 6$ & $266 \pm 100$ & $3 \pm 0.1$ \\
7 & $170 \pm 40$ & $80 \pm 20$ & $83 \pm 25$ & $2.9 \pm 0.1$ \\
8 & $250 \pm 50$ & $165 \pm 16$ & $476 \pm 100$ & $3.4 \pm 0.15$ \\
9 & $300 \pm 60$ & $165 \pm 27$ & $136 \pm 30$ & $0.12 \pm 0.05$ \\
10 & $250 \pm 70$ & $165 \pm 26$ & $200 \pm 65$ & $3.11 \pm 0.04$ \\
11 & $150 \pm 60$ & $170 \pm 14$ & $147 \pm 65$ & 0.3 \\
12 & $150 \pm 50$ & $260 \pm 10$ & $775 \pm 170$ & $3.7 \pm 0.3$ \\
\hline
\end{tabular}

Detection of periods above the cutoff value (Figure 3) suggests that these waves may tunnel through the evanescent region by propagating across inclined magnetic field lines.

The damping properties of the observed transverse oscillations are investigated by fitting the intensity profiles with an exponentially decaying cosine function, $z(t)=$ $A \exp (-t / \tau) \cos (2 \pi t / T+\phi)$, where the fitting parameters $A, T, \tau, \phi$ are the displacement amplitude, oscillation period, damping time, and phase, respectively (Aschwanden et al. 2002). We have found 12 cases where the mottle oscillations show evidence for damping. In Figure 4 we highlight six examples fitted with the above function and the oscillation parameters determined are listed in Table 1. The time sequence is divided into five shorter overlapping sections, and errors are obtained from the maximum and the minimum decay rates and phase of the sections.

In Figure 5, we plot the estimated damping time as a function of the oscillation period. We also calculate a best-fit power-law scaling. The fit indicates an almost linear dependence on the damping time of the kink wave period (Figure 5). Resonant mode conversion predicts that the damping time is a linear function of the period and may therefore be responsible for

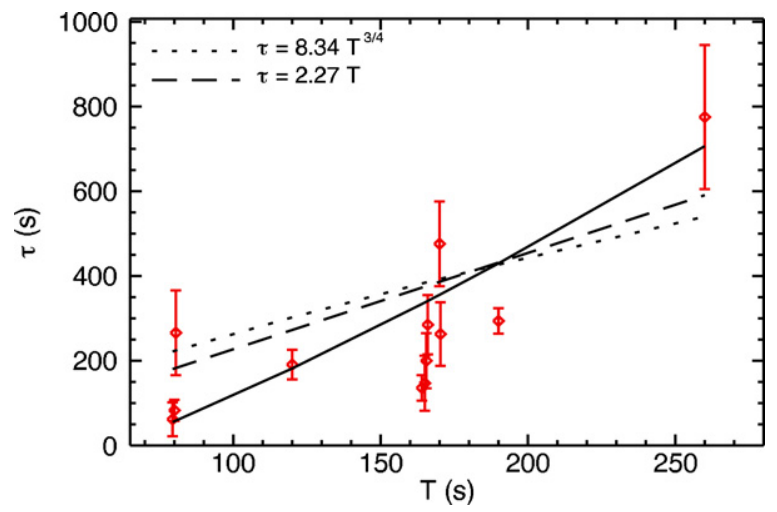

Figure 5. Damping time $\tau$ vs. period $T$ (red diamonds). The solid line is the best-fit scaling law. The dashed line is the fit to $\tau=c_{1} T$ (scaling for resonant absorption) with $c_{1}=2.27$ and dotted line is the fit to $\tau=c_{2} T^{3 / 4}$ (scaling for phase mixing) with $c_{2}=8.34$.

(A color version of this figure is available in the online journal.)

the damping of the oscillations (Goossens et al. 2002; Terradas et al. 2010; Soler et al. 2011) in the chromosphere. Phase mixing follows a scaling law of $\tau \sim T^{3 / 4}$ (Figure 5) that can also be a plausible theory for the interpretation of the observed damping (Ofman \& Aschwanden 2002; Mendoza-Briceño et al. 2004). In contrast, a number of the transverse waves show an increase in amplitude with time. This suggests the possibility of a continuous driver or the influence of a time-dependent plasma (Morton \& Erdélyi 2009; Ruderman 2011). At present, it is not possible to determine which, if any, of these options is responsible for the increasing amplitude.

\section{CONCLUDING REMARKS}

We have presented observations of relatively long-lived dark and bright mottles displaying transverse motions lasting for at least one wave cycle. The mottle oscillations studied in this work are interpreted as fast kink MHD waves. We obtain statistics on the properties, i.e., period, displacement, and velocity amplitudes, of 42 separate events. Coherent oscillations in neighboring mottles indicate an oscillatory source that operates on 
spatial scales larger than the mottle itself. The distance between in-phase oscillating mottles varies between 300 and $1000 \mathrm{~km}$, which is very similar to granular sizes. The relation between the damping times and periods suggests that resonant mode conversion and phase mixing may be viable damping mechanisms (Figure 5). Dissipation of the wave energy associated with the transverse motions is thought to be important for the heating of the quiet solar corona and the acceleration of the solar wind (Cranmer 2007).

Possible excitation mechanisms for the waves include granular buffeting, global oscillations, and reconnection events (Roberts 1979; Spruit 1981; Hollweg 1981; Hasan \& Kalkofen 1999; De Pontieu et al. 2004; He et al. 2009a). Recently, Jess et al. (2012) suggested that longitudinal pressure modes in photospheric magnetic bright points can be converted into the transverse oscillations observed in Type I spicules. Following the successful approach of coronal seismology (for reviews, see, e.g., Ruderman \& Erdélyi 2009; Van Doorsselaere et al. 2009), it may be possible that the observed kink waves could be exploited for seismology of the chromosphere (e.g., Verth et al. 2011). The higher temporal and spatial resolution of ground-based observing instruments (e.g., ROSA/DST, Crisp Imaging SpectroPolarimeter (CRISP)/Swedish Solar Telescope) provides a unique opportunity to study these short-lived, propagating chromospheric waves in detail. Such a study is not possible, at present, for the coronal counterpart (Tomczyk et al. 2007; Erdélyi \& Taroyan 2008).

Observations were obtained at the National Solar Observatory, operated by the Association of Universities for Research in Astronomy, Inc. (AURA) under agreement with the National Science Foundation. R.E. acknowledges M. Kéray for patient encouragement. The authors are also grateful to NSF, Hungary (OTKA, Ref. No. K83133). This work is supported by the UK Science and Technology Facilities Council (STFC), with D.B.J. particularly grateful for the award of an STFC post-doctoral fellowship. We thank the Air Force Office of Scientific Research, Air Force Material Command, USAF for sponsorship under grant number FA8655-09-13085.

\section{REFERENCES}

Aschwanden, M. J., de Pontieu, B., Schrijver, C. J., \& Title, A. M. 2002, Sol. Phys., 206, 99

Banos, G. J., \& Macris, C. J. 1970, Sol. Phys., 12, 106

Beckers, J. M. 1968, Sol. Phys., 3, 367

Beckers, J. M. 1972, ARA\&A, 10, 73

Bray, R. J. 1969, Sol. Phys., 10, 63

Bray, R. J., \& Loughhead, R. E. 1974, The Solar Chromosphere (London: Chapman and Hall)

Cranmer, S. R., van Ballegooijen, A. A., \& Edgar, R. J. 2007, ApJS, 171, 520

De Pontieu, B., Erdélyi, R., \& James, S. P. 2004, Nature, 430, 536

De Pontieu, B., McIntosh, S. W., Carlsson, M., et al. 2007, Science, 318, 1574
Edwin, P. M., \& Roberts, B. 1983, Sol. Phys., 88, 179

Erdélyi, R. 2006a, Phil. Trans. R. Soc. A, 364, 351

Erdélyi, R. 2006b, in Proc. SOHO 18/GONG 2006/HELAS I, Beyond the Spherical Sun, ed. K. Fletcher \& M. J. Thompson (ESA SP-624; Noordwijk: ESA), 15

Erdélyi, R., \& Fedun, V. 2007, Science, 318, 1572

Erdélyi, R., \& Taroyan, Y. 2008, A\&A, 489, L49

Goossens, M., Andries, J., \& Aschwanden, M. J. 2002, A\&A, 394, L39

Guglielmino, S. L., Bellot Rubio, L. R., Zuccarello, F., et al. 2010, ApJ, 724, 1083

He, J., Marsch, E., Tu, C., \& Tian, H. 2009a, ApJ, 705, L217

He, J., Tu, C., Marsch, E., et al. 2009b, A\&A, 497, 525

Hansteen, V. H., De Pontieu, B., Rouppe van der Voort, L., van Noort, M., \& Carlsson, M. 2006, ApJ, 647, L73

Hasan, S. S., \& Kalkofen, W. 1999, ApJ, 519, 899

Hollweg, J. V. 1981, Sol. Phys., 70, 25

Jess, D. B., Mathioudakis, M., Christian, D. J., et al. 2010, Sol. Phys., 261, 363

Jess, D. B., Pascoe, D. J., Christian, D. J., et al. 2012, ApJ, 744, L5

Kim, Y., Bong, S., Park, Y., et al. 2008, J. Korean Astron. Soc., 41, 173

Kukhianidze, V., Zaqarashvili, T. V., \& Khutsishvili, E. 2006, A\&A, 449, L35

Kuridze, D., Mathioudakis, M., Jess, D. B., et al. 2011, A\&A, 533, 76

Mendoza-Briceño, C. A., Erdélyi, R., \& Sigalotti, L. D. G. 2004, ApJ, 605, 493

Morton, R. J., \& Erdélyi, R. 2009, ApJ, 707, 750

Ofman, L., \& Aschwanden, M. J. 2002, ApJ, 576, L153

Okamoto, T. J., \& de Pontieu, B. 2011, ApJ, 736, L24

Pietarila, A., Aznar Cuadrado, R., Hirzberger, J., \& Solanki, S. K. 2011, ApJ, 739, 92

Rae, I. C., \& Roberts, B. 1982, ApJ, 256, 761

Roberts, B. 1979, Sol. Phys., 61, 23

Roberts, B. 2004, in Proc. SOHO 13 Waves, Oscillations and Small-Scale Transients Events in the Solar Atmosphere: Joint View from SOHO and TRACE, ed. R. Erdélyi, J. Ballester, \& B. Fleck (ESA SP-547; Paris: ESA), 1

Rouppe van der Voort, L., De Pontieu, B., Hansteen, V., Carlsson, M., \& van Noort, M. 2007, ApJ, 660, L169

Rouppe van der Voort, L., Leenaarts, J., de Pontieu, B., Carlsson, M., \& Vissers, G. 2009, ApJ, 705, 272

Ruderman, M. S. 2011, Sol. Phys., 271, 41

Ruderman, M. S., \& Erdélyi, R. 2009, Space Sci. Rev., 149, 199

Soler, R., Terradas, J., Verth, G., \& Goossens, M. 2011, ApJ, 736, 10

Spruit, H. C. 1981, A\&A, 98, 155

Spruit, H. C., \& Roberts, H. 1983, Nature, 304, 401

Sterling, A. C. 2000, Sol. Phys., 196, 79

Sterling, A. C., Shibata, K., \& Mariska, J. T. 1993, ApJ, 407, 778

Tavabi, E., Koutchmy, S., \& Ajabshirizadeh, A. 2011, New Astron., 16, 296

Terradas, J., Goossens, M., \& Verth, G. 2010, A\&A, 524, A23

Tomczyk, S., McIntosh, S. W., Keil, S. L., et al. 2007, Science, 317, 1192

Torrence, C., \& Compo, G. P. 1998, Bull. Am. Meteorol. Soc., 79, 61

Trujillo Bueno, J., Merenda, L., Centeno, R., Collados, M., \& Landi DeglInnocenti, E. 2005, ApJ, 619, L191

Tsiropoula, G., \& Schmieder, B. 1997, A\&A, 324, 1183

Tsiropoula, G., \& Tziotziou, K. 2004, A\&A, 424, 279

Van Doorsselaere, T., Verwichte, E., \& Terradas, J. 2009, Space Sci. Rev., 149, 299

van Noort, M., \& Rouppe van der Voort, L. 2006, ApJ, 648, L67

Verth, G., Goossens, M., \& He, J.-S. 2011, ApJ, 733, L15

Wöger, F., von der Lühe, O., \& Reardon, K. 2008, A\&A, 488, 375

Zachariadis, Th. G., Dara, H. C., Alissandrakis, C. E., Koutchmy, S., \& Contikakis, C. 2001, Sol. Phys., 202, 41

Zaqarashvili, T. V., \& Erdélyi, R. 2009, Space Sci. Rev., 149, 355

Zaqarashvili, T. V., Khutsishvili, E., Kukhianidze, V., \& Ramishvili, G. 2007, A\&A, 474, 627 